\title{
Protection of the lung from blast overpressure by stress wave decouplers, buffer plates or sandwich panels
}

\author{
Andrew Sedman, A Hepper
}

Platform Systems Division, Defence Science and Technology Laboratory, Salisbury, UK

\section{Correspondence to} Dr Andrew Sedman, Defence Science and Technology Laboratory, Platform Systems Division Salisbury, Wiltshire, UK; ajsedman@dstl.gov.uk

Received 13 November 2017 Revised 15 January 2018 Accepted 17 January 2018 Published Online First 19 March 2018
Check for updates

To cite: Sedman A, Hepper A. I R Army Med Corps 2019:165:22-26.

\section{ABSTRACT}

Introduction This paper outlines aspects of UK Ministry of Defence's research and development of blast overpressure protection technologies appropriate for use in body armour, with the aim of both propagating new knowledge and updating existing information.

Methods Two simple models are introduced not only to focus the description of the mechanism by which the lungs can be protected, but also to provide a bridge between fields of research that may hold the key to further advances in protection technology and related body armour.

Results Protection can be provided to the lungs by decoupling the stress wave transmission into the thorax by managing the blast energy imparted through the protection system.

Conclusions It is proposed that the utility of the existing 'simple decoupler' blast overpressure protection is reviewed in light of recent developments in the treatment of those sustaining both overpressure and fragment injuries. It is anticipated that further advances in protection technology may be generated by those working in other fields on the analogous technologies of 'buffer plates' and 'sandwich panels'.

\section{INTRODUCTION}

While penetrating injuries pose the greatest threat for people in the vicinity of explosions, protection from blast overpressure injury has been considered important for individuals undertaking specific roles or shielded from projectiles due to body armour or other barriers. Aside from hearing protection, work on developing personal protection from the direct effects of blast overpressure has focused on mitigating injuries caused to the lungs by the short duration blasts. Such short duration blasts have a positive phase duration in the order of a few milliseconds and can be generated by conventional munitions or improvised explosive devices. They may result in primary blast injuries in which the direct coupling of the blast wave into the thorax is considered the principal injury mechanism. ${ }^{1}$ This is in comparison to the gross thoracic deformation associated with the long duration blast loadings produced in the open by very large explosive detonations or produced in specific environments that focus the blast wind, such as tunnels.

The coupling of the blast wave into the thorax, with subsequent stress wave propagation and distortions of the lung tissue, can cause pathological features observable at the macroscopic level, as well

\section{Key messages}

Lungs can be protected from blast overpressure by a simple decoupler.

- Protection requires both stress wave decoupling and the management of energy in the protection.

- Migrating protection technology into effective protection requires a systems approach.

- Advances in the understanding and treatment of blast injuries merit a review of the benefits of the available protection technology.

- Further developments in personal blast protection may arise from research in analogous fields.

as via light and electron microscopes. Light microscope observations include blood-filled alveoli and small airways, as well as disruption of the alveolar walls with bleeding from torn capillaries. ${ }^{2}$ However, electron microscopy indicates damage may be more widespread, with histopathological changes observed in regions without such bleeding. ${ }^{3}$ While aspects of the damage mechanism are yet to be determined, managing the energy transferred to the lung tissue can mitigate such injury.

The UK Ministry of Defence's (MOD's) research and development of blast overpressure protection technologies appropriate for use in body armour has been driven by requirements. Work in the 1980s and 1990s focused on improving the survivability of individuals dressed in explosive ordnance disposal (EOD) suits. ${ }^{14}$ The next peak in research activity aimed to provide mitigation of the emerging blast weapon threat. ${ }^{56}$ More recently, interest in providing such protection to particular user communities by modifying their ballistic plates has revived interest in this research. The aim of this paper is to describe the UK MOD's development of personal protection from blast overpressure injury to the lungs. It suggests reviewing the benefits of such technology in the light of changing protection priorities and updates the institutional memory on primary blast protection methods.

\section{METHOD}

The UK MOD's personal blast overpressure protection research spans a number of decades, and it is not possible to report all aspects of that research here. Instead, two simple models are used to explain the key features of the protection technology. Work 


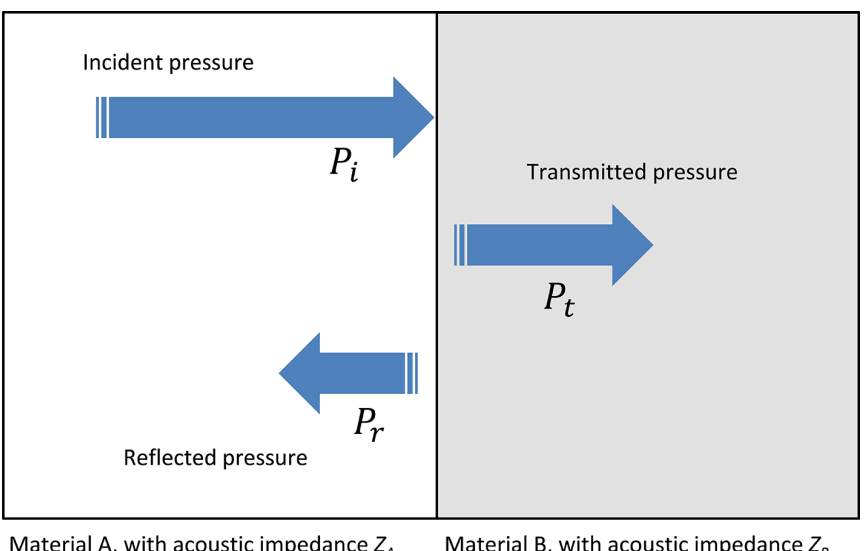

Figure 1 Schematic of a pressure wave's interactions at a boundary between two materials, $A$ and $B$.

on the first of these was published in the $1990 \mathrm{~s},{ }^{178}$ but there is a lack of open publications on the UK MOD's use of the second model. To address this gap, analogous fields of research reported in the more recent open scientific literature are drawn on. This paper cites that research to strengthen the link between these fields and initiate a greater dialogue between them; potentially leading to improvements in protection technology.

\section{RESULTS}

Metrics for injury from short duration blast overpressure

Cooper and Jönsson ${ }^{1}$ attributed the initial instigation of lung injury from short duration blast to the coupling of the incident shock wave into the thorax, noting that this occurs via the initial rapid acceleration of the thoracic wall. For short duration blast loading, both the velocity and the displacement associated with that acceleration are small in magnitude. A relationship between the acceleration of the thoracic wall of terminally anaesthetised pigs and the severity of lung injury was established for animals exposed to short duration blast loading generated by either an explosively driven shock tube or directly by explosives ${ }^{19}$; this relationship suggested that lung injury occurred when the body wall acceleration was $>10 \mathrm{~km} \mathrm{~s}^{-2}$.

The relationship between the motion of the body wall and the severity of lung injury underpinned the development of the Simple Thoracic Rig. ${ }^{10}$ This is a physical model that simulates the peak acceleration of the pig's body wall under simple blast loading and thereby provides estimates of the likely severity of lung injury. In a development of that model, the Enhanced Thoracic Rig was produced, in which the motion of the rig wall is recorded at eight locations to enable estimates of injury from more complex blast loadings, ${ }^{11}{ }^{12}$ such as those in and around buildings.

\section{Protection technology considered as the stress wave decoupler}

As part of the research to improve the survivability of those using EOD suits, a technology that mitigated lung injury from blast overpressure injury was developed by the UK MOD in the 1990s. ${ }^{13}$ In practical terms, injury mitigation was provided by the insertion of a layer of foam behind the existing hard plate of the EOD suit. ${ }^{1}$ Experimental studies demonstrated the performance of this technology in reducing the severity of lung injury of terminally anaesthetised pigs (assessed by lung weight at post mortem). ${ }^{149}$ The injury metric used, Qi, was the ratio of the weight of exposed lungs to the predicted normal lung weight for the animal. A Qi value of 1 implies uninjured lungs, whereas a Qi of 2 signifies lungs that are twice as heavy as normal; a severe injury with a high risk of mortality. ${ }^{9}$ The protection performance was such that the insertion of the appropriate plate and foam combination reduced injury where the Qi was in the region of 1.7 to a level that was within the spread of unexposed lungs. ${ }^{19}$ The use of a plate alone was ineffective at reducing the lung injury. ${ }^{9}$ It was also found that the use of the foam alone increased the injury severity. ${ }^{7}$

Due to the understanding of the protection mechanism, and the explanatory and explorative models used in the 1990s, ${ }^{19}$ this technology was considered to be a stress wave decoupler, and when constructed from a single-front layer (ballistic plate) and monolithic foam backing was referred to as a 'simple decoupler'. This decoupling behaviour was primarily explained using an acoustic transmission model, ${ }^{17-9}$ which is illustrated graphically in Figure 1.

The rationale underpinning this model was that when a compressive stress wave in a material encounters an interface with a second material the amplitudes of the reflected and the transmitted waves depend on the relative characteristic acoustic impedances of the materials. The characteristic acoustic impedance $(\mathrm{Z})$ is the product of the speed of sound in the material and its density. For conventional engineering materials, it can also be related to the material's Young's modulus (E) and its density $(\rho)$ via Equation $1 .^{1}$

$$
Z=(E \times \rho)^{0.5}
$$

For a one-dimensional acoustic wave of pressure amplitude $P_{i}$ travelling through material $\mathrm{A}$, incident on a boundary with material $\mathrm{B}$, the amplitude of the transmitted wave $\left(P_{t}\right)$ and reflected wave $\left(P_{r}\right)$ are given by Equations 2 and 3, respectively. ${ }^{1}$

$$
\begin{gathered}
P_{t}=P_{i} \times\left(\frac{2 \times Z_{B}}{Z_{A}+Z_{B}}\right) \\
P_{r}=P_{t}-P_{i}
\end{gathered}
$$

When applying the acoustic transmission model to the simple decoupler, material A was the ballistic plate and material B the foam backing. While more complex acoustic models were used to predict the amount of stress wave attenuation at different frequencies especially when using viscoelastic foams, the simple acoustic transmission model influenced the early understanding, and development, of blast protection. The key issue was seen as maximising the difference between $Z_{A}$ and $Z_{B}\left(Z_{A}>>Z_{B}\right)$. This influenced the statements given, at that time, that an ideal backing material should be highly compliant (thus low Young's modulus) and have a large air content. ${ }^{1}$

The acoustic transmission model appeared to explain how the protection technology worked. However, there were some aspects that it did not explain. That model took no account of the thickness of the materials, only the interface between them. Nor did it fully explain the results of practical tests, which indicated that increasing the mass of the high impedance layer, or increasing the foam thickness, resulted in lower injury predictions. ${ }^{5}$ In the 1990 s, there was greater recognition that the later time dynamic response of the protection also needed to be accounted for, and explanations found for what was happening when the protection could be overmatched. A simple representation of a mechanical decoupler using a mass (the ballistic plate) on a viscoelastic spring (the foam) provided further insight. 


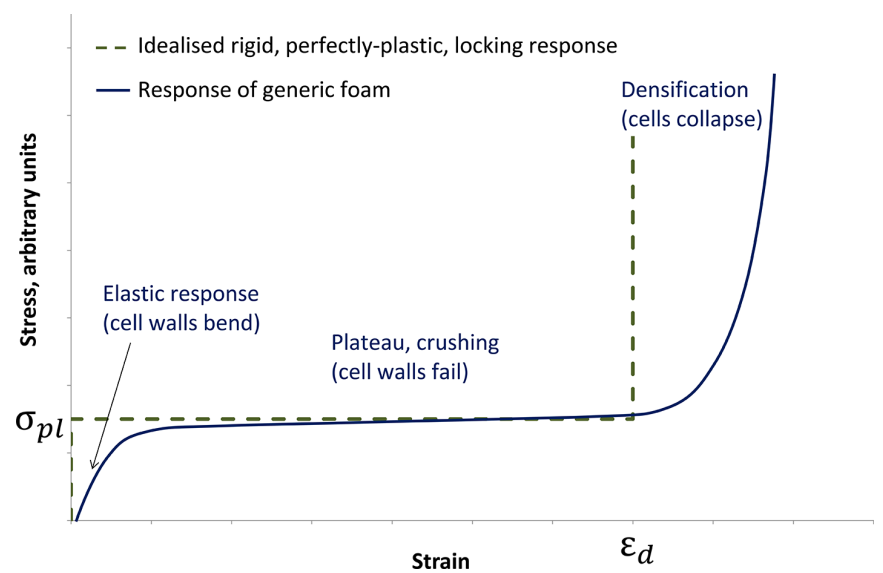

Figure 2 Schematic of the mechanical behaviour of a generic foam under compressive loading and an idealisation of it.

\section{Protection technology considered as a buffer plate}

When subjected to a blast overpressure loading, after the initial stress wave interactions, the ballistic plate within a simple decoupler moves towards the body, having acquired a momentum that is equal to the impulse transmitted to it from the blast loading. Tests have shown that plates can acquire speeds of several tens of metres per second for a representative blast threat. With no intervening material the plate would impact the thorax and could cause impact injury.

To provide protection with a foam-backed plate the energy possessed by the moving plate has to be managed, ideally bringing it to rest before it compresses the foam backing. For if the foam starts to compact, to the extent of losing its cellular structure and densifying, the load transmitted through it will increase dramatically. Using these principles a simple model can be produced that relates key aspects of the protection technology (such as the plate's areal density, the mechanical response and thickness of the backing foam) to key aspects of the blast loading including its impulse or the ability of the applied pressure to do work on the plate.

The approach of using a mass and compliant backing to manage energy deposition is not unique to the field of personal blast protection, and a number of simple equation-based models are available in the literature. Within those fields the terms 'buffer plate' or 'sandwich panel' are used to describe mechanical systems, which in terms of construction are akin to the simple decoupler described above, especially if the rear face of the sandwich panel is considered as the thoracic wall.

When modifying a ballistic plate to provide blast overpressure protection, the areal density of the plate and the blast threat to be countered are usually defined, so the equations of such simple models are normally arranged to predict the minimum foam thickness required to avoid densification. This requires knowledge of the compressive response of the foam. The main features of such a response are shown in Figure 2 for both a generic foam and a mathematical idealised response. The area under the stress-strain curve equals the energy required to compress the foam.

An example of such a simple model is that provided by Wadley et al, ${ }^{14}$ which relates the minimum foam thickness to the momentum of the plate, the mass of the plate and the mechanical response of an idealised foam (see Figure 2, dotted line), via Equation 4.

$$
T_{\text {min }}=\frac{M^{2}}{2 m_{A} \sigma_{p l} \varepsilon_{d}}
$$

where

$T_{\text {min }}$ is the minimum foam thickness to avoid densification;

$M$ is the momentum (per unit area) of the ballistic plate;

$m_{A}$ is the mass (per unit area) of the ballistic plate;

$\sigma_{p l}$ is the plateau stress (see Figure 2) and

$\varepsilon_{d}$ is the densification strain (see Figure 2).

By linking the model of the protection to one that estimates the blast loading, ${ }^{15}$ it is possible to examine the effect of changing the values of the different protection design parameters in relation to threat aspects such as charge size and distance. Illustrative examples, representing idealised rather than actual protection systems, are shown in Figures 3 and 4.

If the densification strain can be increased, more energy will be absorbed before the load increases, so thinner foam can be used (Figure 4). By the same argument a lower minimum foam thickness would also be predicted if the plateau stress was increased. However, a key tenet of the simple model is that the plateau section of the stress-strain response of the foam limits the load transmitted to the thorax. This imposes a constraint on the materials that can be used; if they are too stiff, sufficient load will be transmitted that results in injury.

\section{DISCUSSION}

The simple decoupler's ability to protect the lung from blast overpressure injury has been demonstrated, and the mechanism by which it protects is now well understood. The simple models presented above (Equations 2 and 4) provide a reasonable level of explanation and insight; however, they have only a limited utility in designing such protective systems.

There are many complexities that these simple models do not address. A significant limitation is that they are one-dimensional; they take no account of issues such as the curvature, coverage or flexibility of a real protection system. Coverage is crucial; inserting the appropriate foam behind an existing ballistic plate may provide some mitigation of lung injury, but the extent of that mitigation is also dependent on the area of the body to which it is applied. The coverage requirements of ballistic and blast overpressure protection are not aligned; this limits the benefits of such a retrospective appliqué approach to blast overpressure protection. Early integration of both technologies into a systems-based approach to personal protection design can avoid such issues.

There are also subtleties within the response of backing foams under the rapid loading associated with blast overpressure; not only does the range of plate velocities mean that the rate dependence of the backing material needs to be considered, but there is the potential for the plate to compress the foam at a rate that is faster than the speed of sound within the material. In simple terms, foam at the front face is compacting before that at the rear face starts to respond. ${ }^{16}$ At such speeds the behaviour of materials can become more heavily influenced by the material's mass and inertia. Understanding this behaviour requires not only more complex models, such as finite element modelling and other computer-based numerical approaches, but a variety of models addressing the problem at different length scales, from the full system, down to the details of deformations within the cell walls of the foam or pressure differentials across alveoli walls of the lung. While this paper focuses on monolithic foam as a backing material due to its use in current protection systems, novel material and structural methods (such as layered and shaped foams, viscoelastic or auxetic materials, three-dimensional weave 


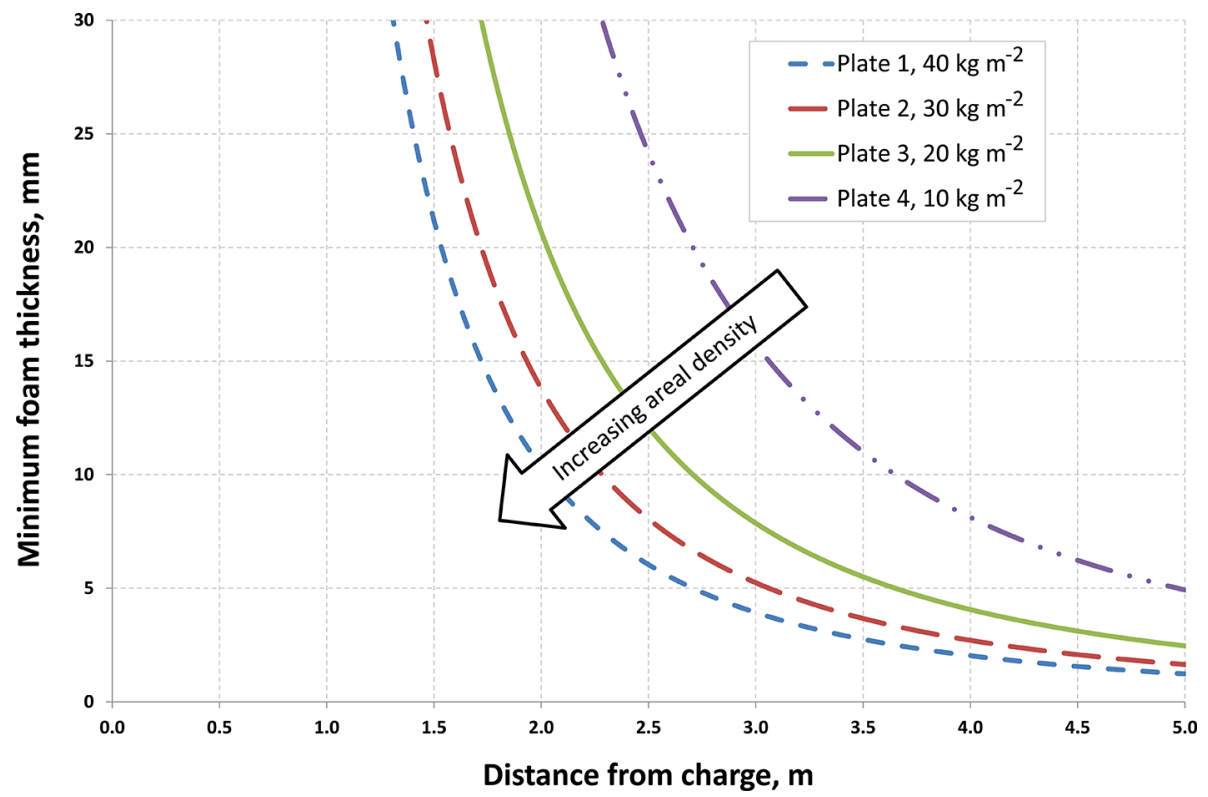

Figure 3 Relationship of minimum foam thickness to distance for a generic foam and representative blast threat, showing the effect of changing the areal density of the ballistic plate all other parameters are fixed.

fabrics or hydroentangled fabrics) to manage the energy deposition may show potential. Some of these approaches will require new models to explain their behaviour.

While protection performance has been demonstrated using terminally anaesthetised pigs and increasingly by using physical surrogates, such as the Enhanced Thoracic Rig, and performance has been predicted using numerical modelling, the performance has been expressed in relation to the mechanical damage of lung tissue. The metrics used have their route either in Qi or the rate of pressure change within discretised regions of lungs. These metrics do not, implicitly, provide any information on physiological impact of lung damage. Although some work on the influence of blast overpressure protection on the arterial partial pressure of oxygen has been published, ${ }^{17}$ little else is available on such protection's influence on pertinent physiological measures. Although benefits were demonstrated in the original animal models, the metrics of protection performance currently used do not provide any indication of the protection offered to other thoracic organs.

The original research on blast overpressure protection was just that, it only addressed primary blast injury. Through the use of animal models ${ }^{18} 19$ significant advances have been made in understanding the physiological interactions of both a blast overpressure injury and haemorrhage. The latter is likely a consequence of penetrating projectiles or secondary blast effects. That research has led to advances in the treatment of those with both blast and haemorrhage. ${ }^{20}$ It may be time to re-evaluate the benefits of blast overpressure protection of the lungs and the metrics used; not in isolation but with a view to understanding how such technology could potentially 'decouple' some of the physiological interactions of those subjected to both blast overpressure and fragment insults.

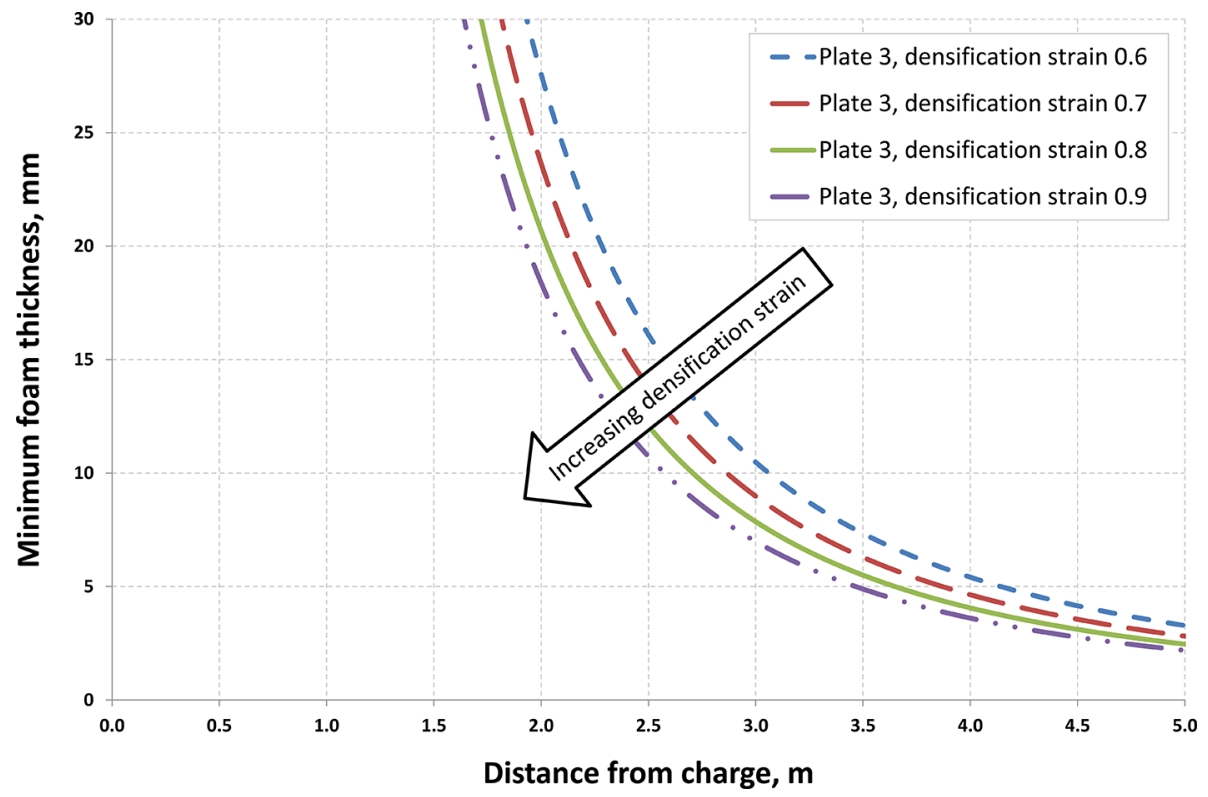

Figure 4 Relationship of minimum foam thickness to distance for a generic foam and representative blast threat, showing the effect of changing the densification strain of the foam for plate $3\left(20 \mathrm{~kg} \mathrm{~m}^{-2}\right)$ in Figure 3, all other parameters are fixed. 
While the work described here was focused on modifying existing ballistic protection and measuring the potential performance of the technology, such performance measures do not directly equate to effective protection. For example, when the technology is incorporated into a body armour system other issues such as the weight and bulk placed on the user and their effect on agility or mobility need to be considered. As demonstrated above, both weight (of the plate) and bulk (of the backing) are positively related to protection: as they increase, protection increases. To migrate this protection technology into a protection system in which the user can effectively perform their military role requires an appropriate balance between these (and other) features. Due to the current state of the art, clinical impacts of such protection cannot be accounted in this balance.

While great progress has been made in recent decades on developing blast overpressure protection for the lungs, many of the significant advances were made by the early workers who referred to stress wave decoupling technology. Current advances being made by researchers in other fields working on buffer plates and sandwich panels, potentially with novel backing materials, may hold the key to future advances in protection. However, the different nomenclature may be limiting awareness that these are two approaches to the same problem. For example, the practical demonstrations of this technology ${ }^{1489}$ are not cited in a more technical treatment of a similar protection technology by Rahimzadeh ${ }^{21}$ that theorises the possibility of providing personal blast protection (although of the head). It is hoped that this paper, by adding to the limited numbers of texts such as that by Wadley et $a l^{14}$ that offer a bridge between these two fields, will not only contribute to the corporate memory but will also stimulate to the exploitation of wider research to provide improved protection for personnel that may face a blast threat in the future.

\section{CONCLUSION}

An overview of some aspects of the blast overpressure protection technology invented and developed by the UK MOD has been presented. While the technology provides significant levels of protection, it is proposed that this performance should be reviewed in light of more recent research and development as well as considering the clinical significance of such injuries. Two simple models have been used to explain the key features of the protection mechanism and some of the deeper complexities of protection design touched on. While it is recognised that the most dramatic advance in protection may always be the initial invention of the 'simple decoupler' in the 1990s by Cooper et $a l^{13}$ it is anticipated that further developments will be made, and potentially by those working in other fields on the analogous technologies of 'buffer plates' and 'sandwich panels'.

Correction notice Since this paper was first published online, an update has been made to the original copyright statement.
Contributors The authors were involved with the original and/or subsequent work described, in either its execution or oversight.

Funding This paper and the UK MOD research described, as funded by UK MOD.

Competing interests None declared.

Provenance and peer review Not commissioned; externally peer reviewed.

(C) Crown copyright (2018), Dstl. This material is licensed under the terms of the Open Government Licence except where otherwise stated. To view this licence, visit http://www.nationalarchives.gov.uk/doc/open-government-licence/version/3 or write to the Information Policy Team, The National Archives, Kew, London TW9 4DU, or email: psi@nationalarchives.gsi.gov.uk.

\section{REFERENCES}

1 Cooper G, Jönsson A. et al Protection against blast injury. In: Cooper GJ, Dudley HAF, Gann DS, Little RA, Maynard RL, . eds. Scientific foundations of trauma. New York, NY: Butterworth-Heinemann, 1997:258-83.

2 Maynard RL, Coppel DL, Lowry KG. et al Blast injury of the lung. In: Cooper GJ, Dudley HAF, Gann DS, Little RA, Maynard RL, . eds. Scientific foundations of trauma. New York, NY: Butterworth-Heinemann, 1997:214-24.

3 Brown RF, Cooper GJ, Maynard RL. The ultrastructure of rat lung following acute primary blast injury. Int J Exp Pathol 1993;74:151-62.

4 Cooper GJ, Sedman AJ, Pearce BP. Protection from primary blast effects - general concept, optimisation and model systems. Colchester, UK: Personal Armour Systems Symposium, 1994.

5 Dearden P. New blast weapons. J R Army Med Corps 2001;147:80-6.

6 Dearden P, Cooper G, Sedman A. Thoracic injury rig - a model for developing protection for an emerging blast threat. The Hague, The Netherlands: Personal Armour Systems Symposium, 2002.

7 Cooper GJ, Pearce BP, Cater SR, et al. Augmentation by foam materials of lung injury produced by blast waves. The role of stress waves in thoracic visceral injury at high rates of energy transfer. Proc. IRCOBI 1989;17:123-38.

8 Cooper GJ, Townend DJ, Cater SR, et al. The role of stress waves in thoracic visceral injury from blast loading: modification of stress transmission by foams and highdensity materials. J Biomech 1991;24:273-85.

9 Cooper GJ. Protection of the lung from blast overpressure by thoracic stress wave decouplers. J Trauma 1996;40:105S-10

10 Cooper GJ, Pearce BP, Sedman AJ, et al. Experimental evaluation of a rig to simulate the response of the thorax to blast loading. J Trauma 1996;40:38S-41.

11 Dorn MR, Cooper GJ, Guyott CC. The prediction of primary blast injury in the lungs caused by complex blast loads. San Antonio, Texas: 18th International Symposium on Ballistics, 1999.

12 Cooper G, Sedman A. Thoracic rig simulating the response of pig lung to blast. NATO RTO-TR-017. Reconsideration of the effects of impulse noise: NATO, 2003:All13-15.

13 Cooper GJ, Cater SJ, Townend DJ. Protective clothing, European Patent Office, EP0542769B1, 1996.

14 Wadley HNG, Dharmasena KP, He MY, et al. An active concept for limiting injuries caused by air blasts. Int J Impact Eng 2010;37:317-23.

15 Kingery CN, Bulmash G. Airblast parameters from TNT spherical air burst and hemispherical surface burst. US army ballistic research laboratory technical report ARBRL-TR-02555. Maryland: Aberdeen Proving Ground, 1984.

16 Main JA, Gazonas GA. Uniaxial crushing of sandwich plates under air blast: influence of mass distribution. Int J Solids Struct 2008;45:2297-321.

17 Trimble KT, McLean D, Sedman AJ, et al. Modulation of primary pulmonary blast injury by a new body armour. J Bone Joint Surg Br 2002;84-B:P171.

18 Garner JP, Watts S, Parry C, et al. Development of a large animal model for investigating resuscitation after blast and hemorrhage. World J Surg 2009;33:2194-202.

19 Kirkman E, Watts S, Cooper G. Blast injury research models. Philos Trans $R$ Soc Lond B Biol Sci 2011;366:144-59.

20 Scott TE, Kirkman E, Haque M, et al. Primary blast lung injury - a review. Br J Anaesth 2017;118:311-6.

21 Rahimzadeh T, Arruda EM, Thouless MD. Design of armor for protection against blast and impact. J Mech Phys Solids 2015;85:98-111. 